\title{
Artificial Intelligence in \\ Organizations: New Opportunities \\ for Phenomenon-Based Theorizing
}

\section{Journal Article}

Author(s):

von Krogh, Georg

Publication date:

2018-12-01

Permanent link:

https://doi.org/10.3929/ethz-b-000320207

Rights / license:

In Copyright - Non-Commercial Use Permitted

Originally published in:

Academy of Management Discoveries 4(4), https://doi.org/10.5465/amd.2018.0084 


\title{
ARTIFICIAL INTELLIGENCE IN ORGANIZATIONS: NEW OPPORTUNITIES FOR PHENOMENON-BASED THEORIZING
}

\author{
GEORG VON KROGH ${ }^{1}$ \\ ETH Zurich
}

Artificial intelligence (AI) is a rapidly emerging phenomenon of economic and organizational significance. The article offers a brief introduction to AI in organizations and proposes that the functioning of these systems relevant to organizations, entails task input (data: sound, text, images, and numbers), task processes (algorithms), and task outputs (solutions and decisions). By considering such functions in organizations, new research opportunities emerge in the areas of decision-making and problem-solving. Advancing academic work in these areas is of key importance at this particular moment in time, and should ultimately aim at helping practitioners adopt an informed, prudent, and realistic approach to AI.

\section{THE PHENOMENON}

In private life, we have already domesticated some forms of artificial intelligence (AI) in speech-based assistants, smart cars, drones, or computer games. Yet, AI is more than your average run-of-the-mill technological innovation. Rather than being confined to one or a few applications, $\mathrm{AI}$ is a fundamental, pervasive economic and organizational phenomenon that holds many theoretical challenges and opportunities in store for management scholars (Bamberger, 2018). Today, organizations increasingly use AI across a range of tasks, such as selecting suitable applicants for organizational positions, advising clients on financial products, performing financial transactions, insuring clients, scheduling complex logistics, diagnosing patients and suggesting therapies, forecasting technological development, and tracking down criminal activity. Such tasks are anything but simple-they make great demands on complex search,

I am truly grateful for excellent comments by Peter Bamberger, Shiko Ben-Menahem, Stefano Brusoni, Vivianna Fang He, Florian von Wangenheim, Ce Zhang, and Yash Raj Shresta on earlier version of this manuscript.

${ }^{1}$ Corresponding author. analysis, and reasoning traditionally confined to the realm of human intelligence.

The founding of AI and cognitive science as an academic field dates back to the days of the Macy conferences on human-computer interactions. As this series was about to end, John McCarthy organized a conference at Dartmouth in 1956 that brought together scholars from a variety of academic disciplines such as cybernetics, psychology, and computer science. Marvin Minsky, Herbert Simon, Allan Newell, John McCarthy, and other protagonists laid out an ambitious program for the scientific study of the mind that involved research on computer-based learning, reasoning, problem-solving, and decisionmaking (Roos \& von Krogh, 1995). Although many promises were made on its practical usefulness, AI failed to deliver and the research field lost traction throughout the 1960s and 1970s. During the 1980s and 1990s, governments and firms began making significant investments in research on expert systems, which rejuvenated the interested in AI as did some spectacular events such as IBM's computer software "Deep Blue" winning a game of chess against the grand master Garry Kasparov on May 11, 1997.

Today, the rapid adoption of AI by organizations can be attributed to four major causes. First, the past two decades have seen significant advancements in the science and technology underlying AI methods (e.g., long short-term memory units, recurrent neural networks, and convolutional neural networks). Many companies have made these technologies available under an open-source license (e.g., Google's Tensorflow, Amazon's Alexa, and Microsoft Computational Network Tool Kit), increasing their adoption. Second, information technology has become increasingly efficient at capturing and storing task-related data across the organization. Such data feed the underlying algorithms of AI and are a premise for task automation. Third, although sophisticated AI demands extensive computation, the rapidly decreasing cost of computer hardware and 
AI-dedicated chip designs makes computational power increasingly affordable. Fourth, the growth of cloud-based services in this space has made AI available to different kinds of organizations from start-ups to established firms.

\section{OPPORTUNITIES FOR RESEARCH}

A starting point to explore the opportunities AI offers for phenomenon-based theorizing and abductive reasoning is to consider its functioning in organizations. AI is a broad collection of computer-assisted systems for task performance, including but not limited to machine learning, automated reasoning, knowledge repositories, image recognition, and natural language processing. Such systems entail task input (data: sound, text, images, and numbers), task processes (algorithms), and task outputs (solutions, decisions). When deployed to automating organizational tasks, AI places great demands on the quality of input data (correct labeling, complete data, and detectable noise). Systems need flawless data to learn from experience and feedback from their environment and, thus, improve task performance. An emerging research topic on AI is how systems can efficiently identify and handle many types of noisy data. Yet, at this time the requirement for flawless data input to AI still strongly influences when and where it can be put to use in organizations (e.g., where tasks are repetitive and quality data are generated.)

Although task processes tend to fall within the realm of computer science and mathematics, understanding $\mathrm{AI}$ as an ensemble of algorithms can help management scholars make sense of the opportunities and limitations associated with such systems in empirical situations. Task outputs are often easy to observe, yet understanding and explaining why a particular output came about requires familiarity with the algorithm, human judgment, andinterpretation (Puranam, Shrestha, He, \& von Krogh, 2018).

Finally, AI provides two broad types of task output to organizations. "Decisions" - conclusions reached from algorithmic deliberation based on the data available-and "solutions"-alternative courses of action to resolve a problem. Both need rich datadriven discovery and tentative explanations, as I shall argue below. I first elaborate on abductive reasoning around decision-making in organizations, and then move on to problem-solving, before concluding.

\section{MAKING DECISIONS WITH INTELLIGENT MACHINES}

In anticipation of efficient task outcomes, individuals and organizations delegate decision-making authority to AI, such as when the autopilot makes a car change lanes or a trading algorithm sells a large position of foreign currency on a high-frequency trading platform (Schwarting, Alonso-Mora, \& Rus, 2018). To understand the problem of delegation, it is useful to turn to the Carnegie School of organization theory. Here, deductive reasoning about organization design and decision-making explicitly or implicitly assumes bounded rationality (Levinthal \& Workiewicz, 2018; Puranam, Stieglitz, Osman, \& Pillutla, 2015). Humans have limited capacity for processing information-or consciously ignore information (Feldman \& March, 1981) - which restrains the consideration of decision alternatives. To mitigate this problem, informationprocessing and decision-making authority can be delegated across roles and units that display various degrees of interdependence. The design of horizontal and vertical information structures is a necessary condition to efficiently coordinate decision-making among these roles and units (Aoki, 1986). Common coordination mechanisms, in turn, include goals, hierarchies, rules, and incentives (Galbraith, 1974; March \& Simon, 1958).

Delegating decision-making authority to $\mathrm{AI}$, however, may alter organizations in unprecedented ways. Data flow can be centralized around highthroughput data-processing algorithms (e.g., a trading algorithm) and need no longer follow information structures spanning units and specialist roles occupied by humans (e.g., a team of expert analysts who each deliver decision-relevant input to the trading desk). Such designs resemble what Galbraith (1974) calls a "self-contained task" and curtails the organizations' overall cost information processing brought about by the need to invest in vertical information systems or lateral relations. Moreover, AI may stay programmed on one or more goals and requires no particular incentive to process "all" the information available.

Thus, a fundamental research topic for management scholars is how the phenomenon of AI relates to organization design. In other words, what decision-making authority can or cannot be delegated to intelligent machines (e.g., decisions that require intellectual effort vs. moral judgment) and what are the effects on task performance? In what situations can AI make decisions or merely offer decision support, as was common in the early days of expert systems? How does such delegation alter or disrupt vertical and horizontal information structures and the flow of data in organizations? When and where do organizational members' process input task information to AI? For example, to evolve AI demands feedback from users on the quality of the decision made or suggested. Moreover, what are the considerations of ethics and accountability in 
the delegation of decision-making (rights) to AI (McLaren, 2003)? What are legal implications of so doing for organizations?

Because these and similar questions seem to fall outside of the explanatory domain of much contemporary organization theory rooted in an informationprocessing view, the AI decision-making phenomenon is particularly suitable for abductive reasoning (Bamberger, 2018). An abductive research program should start by collecting quantitative and qualitative data on situations where decision-making authority is delegated to AI. Researchers may describe features of these situations in terms of task input and task processes. Thereby, we can gain deeper understanding of the constraints on AI authority across the organization.

Based on situational facts, researchers can next conceptualize classes of decisions subject to delegation of authority and identify what salient task data feed algorithms. An investigation of task data flows will likely reveal insights on the dynamics of information structures to coordinate information processing in the organization, as well as what coordination mechanisms are in play. It will be important to discover how and under what conditions task input data are generated automatically or rely on human processing and input, and what task processes (algorithms) are deployed. By gradually uncovering AI as a fundamental organizational phenomenon, we may distinguish and offer tentative explanations of the emergence and interaction of human and machine authority regimes in organizations.

Moreover, to better understand and explain the delegation of decision-making authority to AI, it is important to gather qualitative and quantitative data on delegation failure (e.g., is there such a thing as moral hazard by machines?). For example, trading algorithms reduce information-processing costs and decision-making time, but a wrong trading decision may risk the survival of a financial firm in a split second. A medical AI assistant reliably processes complete data and the experience available, but a false diagnosis based on an undetected data flaw may risk a patient's life. Through abductive reasoning, we may better understand what it means to delegate authority to AI in organizations, what the role of human responsibility and accountability is in such delegation, and how organizations, organizational members, and machines learn how to improve AI decision-making in various situations.

A focus on delegation inevitably raises many concerns such as substituting a labor force through AI (Brynjolfsson \& McAfee, 2014) or the undesirable consequences of biases and reinforcement learning in automated decision-making (e.g., discrimination)

\section{SOLVING PROBLEMS WITH INTELLIGENT MACHINES}

Problem-solving and formulationare considered key activities of organizations (Ben-Menahem, von Krogh, Erden, \& Schneider, 2016; Brusoni, 2005; Hargadon \& Bechky, 2006; Miron-Spektor, Ingram, Keller, Smith, \& Lewis, 2018; Nickerson \& Zenger, 2004) and, here too, AI has proven useful by automatically generating solutions (Flasinski, 2016) to to some organizational problems.

Problem formulation is an important condition for diving the labor of problem-solving in organizations (Becker \& Baloff, 1969; see Von Hippel \& von Krogh, 2016: 212), and today, intelligent machines are "laboring" to solve problems formulated within a wide range of domains, including designing, planning, searching, sorting, structuring, etc. AI can be used to design lightweight building components, assemble financial portfolios that fit client needs, have simple conversations with patients, and recommend rerouting cargo in a clogged railway transportation system. Yet, beyond such striking examples, how problem-solving with the involvement of intelligent machines unfolds in organizations remains a poorly understood phenomenon.

The study of problem-solving has occupied a central position in the field of cognitive science and AI because its inception (Newell \& Simon, 1972). According to Herbert Simon (Simon, 1973), problem-solving entails searching for solutions to a formulated problem in an (extensive) solution space, and reasoning that departs from problem formulation and is then applied to information as acquired by the solver. Simon (1973) distinguished well-structured problems that lend themselves to algorithmic search in a solution landscape from ill-structured problems that contain extensive situational data. The latter need to be converted into well-structured problems for algorithmic search (Simon, 1973). Thus, devising efficient problemsolving procedures presupposes an understanding of "real" problem solvers in action (March, 1978). The problem solver supplies the empirical premises that are based on their knowledge of the world. Within their domain of operation, intelligent machines can gain such knowledge too, but hardly beyond it. So far, it remains a human prerogative to juxtapose prior experience to problematic situations and creatively put such experience to use in novel domains. As Simon (1973: 12) concludes "There is no valid substitute for the problem solver knowing what can validly be assumed about the empirical situation in the context of any given problem."

Since Simon's seminal studies, technological advances have enabled contemporary $\mathrm{AI}$ to process 
extensive and different types of situational data and increasingly solve ill-structured problems (e.g., voice recognition, conversational turns, sentiment analysis, image analysis). Yet, the challenge of solving real-world problems in organizations remains unchanged: inferring from data some plausible but tentative explanations or "general" solutions. Therein lies the imperative for phenomenon-based theorizing (Bamberger, 2018) propelled by a guiding question: What constitutes a problematic situation in an organization that benefits or may benefit from solutions generated by AI?

Shedding light on this question can begin by recognizing the principle of division of labor. Although AI may effectively search out optimal solutions in a predefined landscape, people across the organization remain superior in formulating problems worth solving by either humans or systems. Thus, researchers may proceed by exploring deep causes for a problem (Schwenk \& Thomas, 1983), even backtracking to the fundamental needs of the problem solver before their articulation of a "problem statement" (Von Hippel \& von Krogh, 2016). What problem formulations and types of organizational problem-solving involve division of labor between organizational units, members, and AI? What needs and problems provide the impetus for AI involvement in solution generation? Building on a recent finding that people's performance in solving ill-structured problems hinges on their cognitive flexibility or recognition of diversity (Laurerio-Martinez \& Brusoni, 2018) and interesting question emerges: Can AI augment such human problem-solving performance by its extensive capacity at sifting through massive data and discovering valuable candidate solutions?

Ideally, an abductive research program collects qualitative and quantitative data on problematic situations in organizations that are malleable to the use of AI. Yet, as was the case for research on decision-making, it may be expedient to gather data on task input (problem-relevant) and task processes (e.g., search or reasoning algorithms) because such data not only reveals features of, but also indicates constraints on, problematic situations where AI involvement is costly or in other ways difficult. ${ }^{3}$ For example, if you need to fasten your bedside table to the wall, it is (hopefully) a non-repetitive situation where automation is too costly. Another realistic constraint is that AI typically draws on historical data and experience to offer tentative problem solutions. How could this impede the problem solver to generate creative solutions to existing problems and how, if at all, are solvers aware of this constraint? In

${ }^{3}$ An interesting site to start could be call centers where AI assisted problem-solving is common practice. See also MacKinnon Clark, Tan, Murfett, Rogers, and Ang (2018) data-rich and ill-structured problematic situations, why do problem solvers individually and collectively choose not to invest effort in the search for creative solutions, remain docile, and accept AI's problem solutions without further scrutiny? Over time, does such behavior impact on the effectiveness of problem-solving within an organization?

Interpretation and justification of solutions are inherent features in human problem-solving. However, the manner in which interpretation and justification is individually and collectively applied to AI-generated output emerges as concerns for management scholars (a similar argument was made for decision-making by Agarwal, Gans, \& Goldfarb, 2018). Although algorithms offer "solutions," for example, sorting complex data or predicting the likelihood of an event, they remain tentative and in themselves cannot offer an explanation or justification (Paul, 1993). Hence, problem solvers need to interpret and justify the "solutions" offered, by reexamining the data and sense-testing task outcomes in organizations. For example, when using machine learning to analyze large datasets, running alternative algorithms on the same data set is considered good practice as well as offering plausible warrants that connect the data with the conclusions (Puranam et al., 2018).

Consider another example of hospital emergency departments. Sorting "high-risk" from "low-risk" patients is a difficult problem for such departments, and research has found that compared with human operators, machine learning can under some conditions predict faster and better the likelihood that patients who call in will experience cardiac arrest, simply based on the sound and tone of the voice (Blomberg, Folke, Ersboell, \& Lippert, 2018). Yet, treatment includes choices about methods to jumpstart the heart and whether or not to perform surgery. Such choices require experience and physicians' on-site judgment of the patient's condition. In this problematic situation, a "creative assemblage" (Orlikowski, 2007; Suchman, 2007) of team problemsolving and automated solution generation seem to offer the greatest benefits. However, understanding the nature of these assemblages and when and how AI augments task performance in such organizations may require the collection and analysis of rich data on the problematic situation, including how physicians collectively interpret, justify, and ultimately build "trust" in AI solutions.

Finally, with data-driven descriptions of problematic situations in organizations, division of labor, and problem solutions as task output by AI, tentative explanations can be formulated on what emerging patterns of reasoning connect needs, problem statements, and alternative solutions and the alternative 
specification and presentation of an AI-searchable problem.

\section{MOVING AHEAD}

The questions raised previously are just a few written onto a "guidepost"-avant la lettre-for scholarship relevant to the Academy of Management Discoveries. AI has the qualities of being a new but poorly understood phenomenon. By concentrating efforts on collecting quantitative and qualitative data on the aforementionedquestions, we may discover unanticipated relationships and ways to resolve tensions and ambiguities in the research on $\mathrm{AI}$ within the domains organizational decision-making and problem solving. Remarkably, although, when we shift our questions from the realm of decisions to problems, AI suddenly appears to augment rather than substitute said human behavior in organizations. Considering division of labor in problemsolving, organizational members remain superior in problem formulation and perhaps at par with $\mathrm{AI}$ in solution generation, while during AI decisionmaking, there is an underlying and often problematic shift of authority. Ahead, I believe, lies a fertile ground for discovering unexpected combinations of human ingenuity with intelligent machines in organization. Through this endeavor, management scholarship can also make an effort to help practitioners see through all the "hype" and adopt an informed, prudent, and realistic approach to AI. In the long run, outsourcing "intelligence" to machines will neither be useful nor morally right. Although such technologies have many attractive features, they merely emulate cognitive processes and cannot substitute the great flexibility, adaptability, and generativity we associate with human intelligence.

\section{REFERENCES}

Agarwal, A. K., Gans, J., \& Goldfarb, A. 2018. Exploring the impact of artificial intelligence: Prediction versus judgment. NBER. Working paper. 24626.

Aoki, M. 1986. Horizontal vs. vertical information structure of the firm. The American Economic Review, 76: 971-983.

Bamberger, P. A. 2018. Clarifying what we are about and where we are going. Academy of Management Discoveries, $4: 1-10$.

Becker, S. W., \& Baloff, N. 1969. Organizational structure and complex problem solving. Administrative Science Quarterly, 14(2): 260-271.

Ben-Menahem, S. M., von Krogh, G., Erden, Z., \& Schneider, A. 2016. Coordinating knowledge creation in multidisciplinary teams: Evidence from early-stage drug discovery. Academy of Management Joumal, 59(4): 1308-1338.

Blomberg, S. N., Folke, F., Ersboell, A. K., \& Lippert, F. K. 2018. Machine learning model surpassing medical dispatchers' recognition of out-of-hospital cardiac arrest. BMJ Open, 8(Suppl 1): A1-A3.

Brusoni, S. 2005. The limits to specialization: Problem solving and coordination in modular networks. $\mathrm{Or}^{\mathrm{r}}$ ganization Studies, 26(12): 1885-1907.

Brynjolfsson, E., \& McAfee, A. 2014. The second machine age: Work, progress, and prosperity in a time of brilliant technologies. Plassen, NY: WW Norton \& Company.

Feldman, M. S., \& March, J. G. 1981. Information in organizations as signal and symbol. Administrative Science Quarterly, 26(2): 171-186.

Flasinski, M. 2016. Cognitive architectures. In Introduction to artificial intelligence. Cham, Switzerland: Springer.

Galbraith, J. R. 1974. Organization design: An information processing view. Interfaces, 4(3): 28-36.

Hargadon, A. B., \& Bechky, B. A. 2006. When collections of creatives become creative collectives: A field study of problem solving at work. Organization Science, 17(4): 484-500.

Laurerio-Martinez, D., \& Brusoni, S. 2018. Cognitive flexibility and adaptive decision making: evidence from a laboratory study of expert decision makers. Strategic Management Journal, 39(4): 1031-1058.

Levinthal, D. A., \& Workiewicz, M. 2018. When two bosses are better than one: Nearly decomposable systems and organizational adaptation. Organization Science, 29(2): $207-224$

MacKinnon Clark, C., Tan, M. L., Murfett, U. M., Rogers, P. S., \& Ang, S. 2018. The call center agent's performance paradox: A mixed methods study of discourse strategies and paradox resolution. Academy of Management Discoveries, forthcoming.

March, J. G. 1978. Bounded rationality, ambiguity, and the engineering of choice. The Bell Journal of Economics, 9(2): 587-608.

March, J. G., \& Simon, H. A. 1958. Organizations. New York: Wiley.

McLaren, B. M. 2003. Extensionally defining principles and cases in ethics: An AI model. Artificial Intelligence, $150(1-2)$ : 145-181.

Miron-Spektor, E., Ingram, A., Keller, J., Smith, W. K., \& Lewis, M. W. 2018. Microfoundations of organizational paradox: The problem is how we think about the problem. Academy of Management Journal, 61(1): 26-45. 
Newell, A., \& Simon, H. A. 1972. Human problem solving, vol. 104, no. (9). Englewood Cliffs, NJ: Prentice-Hall.

Nickerson, J. A., \& Zenger, T. R. 2004. A knowledge-based theory of the firm-The problem-solving perspective. Organization Science, 15(6): 617-632.

Orlikowski, W. J. 2007. Sociomaterial practices: Exploring technology at work. Organization Studies, 28(9): 1435-1448.

Paul. G. 1993. Approaches to abductive reasoning-An overview. Artificial Intelligence Review, 7(2): 109-152.

Puranam, P., Shrestha, Y. R., He, V. F., \& von Krogh, G. 2018. Algorithmic induction through machine learning: Opportunities for management and organization research. Available at SSRN: https://papers. ssrn.com/sol3/papers.cfin?abstract_id $=3140617$.

Puranam, P., Stieglitz, N., Osman, M., \& Pillutla, M. M. 2015. Modelling bounded rationality in organizations: Progress and prospects. The Academy of Management Annals, 9(1): 337-392.

Roos, J., \& von Krogh, G. 1995. Organizational epistemology. New York: Macmillan and St Martin's Press.

Schwarting, W., Alonso-Mora, J., \& Rus, D. 2018. Planning and decision making for autonomous vehicles. Annual Review of Control, Robotics, and Autonomous Systems, 6(59): 8.1-8.24
Schwenk, C., \& Thomas, H. 1983. Formulating the mess: The role of decision aids in problem formulation. Omega, 11(3): 239-252.

Simon, H. A. 1973. The structure of ill-structured problems. Artificial Intelligence, 4(3-4): 181-201.

Suchman, L. 2007. Human-machine reconfigurations: Plans and situated actions. Cambridge, UK: Cambridge University Press.

Von Hippel, E., \& von Krogh, G. 2016. Identifying viable "need-solution pairs": Problem solving without problem formulation. Organization Science, 27(1): 207-221.

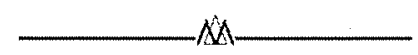

Georg von Krogh (gvkrogh@ethz.ch) is the Professor of Strategic Management and Innovation at ETH Zurich. His research focuses on the knowledge-based view of organizations, innovation, and digital technology. He has published on these topics in Academy of Management Journal, Management Science, Organization Science, Harvard Business Review, MIS Quarterly, Information Systems Research, MIT Sloan Management Review, Research Policy, Strategic Management Journal and other outlets. 